\title{
Optimal Triage for COVID-19 Patients Under Limited Intensive Care Unit Capacity: Development of a Parsimonious Machine Learning Prediction Model and Threshold Optimization Using Discrete-Event Simulation
}

Jeong Min Kim

Korea Advanced Institute of Science and Technology

Hwa Kyung Lim

Korea Advanced Institute of Science and Technology

Jae-Hyeon Ahn

Korea Advanced Institute of Science and Technology

Kyoung Hwa Lee

Yonsei University College of Medicine

Kwang Suk Lee

Yonsei University College of Medicine

Kyo Chul Koo ( gckoo@yuhs.ac)

Yonsei University College of Medicine https://orcid.org/0000-0001-7303-6256

\section{Research}

Keywords: COVID-19, decision support techniques, machine learning, prediction, triage

Posted Date: June 28th, 2021

DOI: https://doi.org/10.21203/rs.3.rs-648718/v1

License: (c) (i) This work is licensed under a Creative Commons Attribution 4.0 International License.

Read Full License 


\section{Abstract}

Background: The coronavirus disease 2019 (COVID-19) pandemic has caused an unprecedented burden on healthcare systems. To effectively triage COVID-19 patients within situations of limited data availability and to explore optimal thresholds to minimize mortality rates while maintaining the healthcare system capacity.

Methods: A nationwide sample of 5601 patients confirmed for COVID-19 up until April 2020 was retrospectively reviewed. XGBoost and logistic regression analysis were used to develop prediction models for the patients' maximum clinical severity during hospitalization, classified according to the WHO Ordinal Scale for Clinical Improvement (OSCl). The recursive feature elimination technique was used to evaluate the extent of the model performance's maintenance when clinical and laboratory variables are eliminated. Using populations based on hypothetical patient influx scenarios, discrete-event simulation was performed to find the optimal threshold within limited resource environments that minimizes mortality rates.

Results: The cross-validated area under the receiver operating characteristics (AUROC) of the baseline XGBoost model that utilized all 37 variables was 0.965 for $\mathrm{OSCI} \geq 6$. Compared to the baseline model's performance, the AUROC of the reduced model that utilized 17 variables was maintained at 0.963 with statistical insignificance. Optimal thresholds were found to minimize mortality rates in a hypothetical patient influx scenario. The benefit of utilizing an optimal triage threshold was clear, reducing mortality up to $18.1 \%$ compared to the conventional Youden Index.

Conclusions: Our adaptive triage model and its threshold optimization capability reveal that COVID-19 management can be integrated using both medical and healthcare management sectors to guarantee maximum treatment efficacy.

\section{Introduction}

The high incidences of critical illness and mortality due to coronavirus disease (COVID-19) have placed unprecedented burdens on healthcare systems. The World Health Organization (WHO) guidelines recommend that all countries should prepare for infection surges in their healthcare facilities, implementing appropriate triage protocols [1]. Unfortunately, these guidelines fail to provide a one-sizefits-all approach that works for individual regions, while accounting for their unique outbreak surges. Until further improvements in treatments and epidemiological immunizations have been achieved, implementing an adaptive triage model is imperative to ensure the most effective utilization of local regions' healthcare resources.

Various triage measures are needed during a pandemic for the lack of established intensive care unit (ICU) resources. First, a reliable prediction model that provides accurate prognoses is needed to facilitate preemptive treatments. This model should not only focus on any particular clinical endpoint but also se spectrums. Second, this prediction model 
should operate using readily available assessment parameters, thereby maintaining its reliability in resource-constrained environments. Third, this model should also consider the availability of ICU at either a facility or national level relative to concurrent patient influx volumes. COVID-19 is associated with disruptions to most healthcare infrastructures. Therefore, an adjustable risk stratification model that considers various regions' ICU availability, as well as one that identifies patients who will likely require intensive care, will help to reduce these systems' burdens.

Prognostic models have been developed to ensure effective triage for COVID-19 patients [2-7]. These models have a modest predictive accuracy; however, their generalizability has been questioned due to their confinement to single clinical outcome measures and reductions in their performance when using insufficient data. Most importantly, these models' classification thresholds, which are crucial for ensuring the effective ICU utilization, have been neglected, thereby limiting their practical implementation capabilities. In sum, these models' shortcomings highlight the need to combine large-scale multiinstitutional data with advanced prediction models, such as those using machine learning and simulation modeling.

Our objectives were three-fold. First, we developed a baseline prediction model with an explanatory feature for triaging COVID-19 patients. Second, we used the recursive feature elimination (RFE) technique to develop reduced models that would help ensure efficient ICU utilization under limited data availability $[8,9]$. Finally, we developed an adaptive triage model using the discrete-event simulation (DES) technique that would assist in efficient ICU utilization under limited resources.

\section{Materials And Methods \\ 2.1. Ethics statement}

This study was approved by an institutional ethics committee (2020-0883-001) and the Korea Disease Control and Prevention Agency (KDCA) epidemiological survey and analysis committee (20201120_4a). All study procedures complied with the 1946 Declaration of Helsinki and its 2008 update.

\subsection{Patient cohort}

Demographic, clinical, laboratory, and disease outcome records of 5628 patients, confirmed for severe acute respiratory syndrome coronavirus 2 up until April 2020, were retrospectively reviewed. Data were comprehensively managed by the KDCA. The database included patients who had been treated and released from quarantine or hospitalization, and those who died from the sequela of COVID-19. Among 5628 patients, 27 patients with missing clinical severity data were excluded, resulting in a final development cohort of 5601 .

\subsection{Covariates and outcome definitions}

Baseline data collected at diagnosis were used for the model development. Demographic data included 
index (BMI). Medical comorbidities included hypertension, diabetes mellitus, heart failure, cardiovascular disease, asthma, chronic kidney disease, chronic obstructive pulmonary disease, chronic liver disease, autoimmune disease, dementia, malignancy, and pregnancy. Clinical findings included history of fever ( $\geq$ $37.5^{\circ} \mathrm{C}$ ), cough, sputum production, myalgia, fatigue, sore throat, rhinorrhea, dyspnea, vomiting, nausea, diarrhea, headache, and altered consciousness. Laboratory data included hemoglobin, hematocrit, white blood cell count, \%leukocyte, and platelet count. The patients' maximum clinical severity during quarantine or hospitalization were classified according to the WHO Ordinal Scale for Clinical Improvement (OSCl) [10].

\subsection{Statistical analysis}

\subsubsection{Model development}

Multivariate logistic regression (LR) and extreme gradient boosting (XGBoost) were used to select the best-performing prediction model [11]. The models were developed and cross-validated using 5037 (89.9\%) patient data and were then revalidated using a 564 (10.1\%) hold-out cohort. Performance metrics were calculated using 10-fold cross-validation. Model development was performed using the caret package in R Model.

\subsubsection{Variable elimination}

RFE was performed for two full models: $\mathrm{F}+\mathrm{L}+$ (with laboratory data) and $\mathrm{F}+\mathrm{L}-$ (excluding laboratory data). Shapley additive explanations (SHAP) was used to rank each variable based on its significance to the model [12]. At each RFE iteration, the lowest-ranked feature was eliminated, the model was refitted, and its performance was assessed. The reduced models (F-L + and F-L-) were then selected at a point wherein the number of features was minimized while the differences in the AUROC remained statistically insignificant. Analysis was performed using caret and SHAPforXGBOOST package in R.

\subsubsection{Model interpretation and comparison}

To interpret the $\mathrm{F}+\mathrm{L}+$ model, we used SHAP as it provides visible post-hoc interpretability to black-box machine learning models [12]. Patient-specific plots were created by aggregating the SHAP score of each variable.

Hyperparameters of the XGBoost algorithm were optimized to maximize its area under the receiver operating characteristics (AUROC) value using a simple grid search. Accuracy, AUROC, sensitivity, positive predicted value (PPV), and negative predicted value (NPV) were calculated at a $90 \%$ specificity using the pROC package in R. Confidence intervals (Cls) of the performance measures were then calculated using a stratified bootstrap method with 2000 replicates.

\subsubsection{Threshold optimization \\ 2.4.4.1. DES and patient influx generation}


We conducted DES to find the optimal threshold that occurs within limited ICU capacity that minimizes mortality rates, as calculated by $\frac{n \text { (totaldeaths })}{n \text { (totalpatients })}$, using the simmer R package.

A hypothetical patient influx scenario was created using the SIR model [13]. The total population calculated was fixed at 60,000 , considering that the largest historical influx observed in South Korea (58654 cumulative patients: November 132020 - February 20 2021). I(0) and R(0) were fixed at six and zero, respectively. The recovery rate gamma was set at 0.05 because the average COVID-19 recovery time was 20.1 days [14]. The transmission rate beta ranged between 0.75 and 5 when generating influxes with different R0 (base reproduction rate) levels. The number of newly confirmed patients per day was obtained from the SIR modeling data (Fig. 1).

\subsubsection{Probability generation}

Out-of-fold prediction results of the 10 -fold cross-validation were aggregated to generate an empirical probability distribution of the disease severity probability. We used the $\mathrm{F}+\mathrm{L}$ - model because of its high performance and its potential use wherein there are limited diagnostic tools. Inverse transformation sampling was performed on the empirical probability distribution function, which was approximated using the Gaussian kernel density estimation and linear interpolation [15]. The process was performed separately for severe and non-severe patients, with the sampled probabilities being randomly matched with the generated patient influx rates while maintaining the prevalence of severe patients. The prediction probability distributions of the out-of-fold and generated samples are presented in Fig. 2.

\subsubsection{Simulation scenarios}

Patients with a severe disease probability above the threshold are directed to the ICU, depending on its current capacity. Rejected patients are then directed to the general ward along with those who have a severe disease probability below the threshold. The probability of severe disease patients dying while in the ICU was 0.507 , while it was 0.990 for those outside of the ICU [16]. Non-severe patients were assumed to survive regardless of ICU admission. Patient deaths were categorized into three types: resourceindependent deaths, wherein severe patients expire despite ICU care (Type I); resource-dependent deaths in which severe patients expired due to ICU unavailability (Type II); and threshold-dependent deaths wherein severe patients expire after being incorrectly classified as "non-severe" and are subsequently directed to the general ward (Type III).

The maximum capacity of the ICU was established as 504 beds based on the number of isolation beds under negative pressure [17]. To estimate the distribution of length of stay, we used a previously suggested gamma distribution with a shape parameter of 1.5488 and a rate parameter of 0.1331 for those who expired, with a shape parameter of 0.8904 and a rate parameter of 0.0477 for those who survived to approximate the median and interquartile range [16, 18]. Simulations were repeated 20 times for each influx scenario to ensure robustness. 


\subsection{Patient characteristics}

Descriptive characteristics of the training and hold-out cohorts are provided in Additional file 1: Table S1. A total of $5330(95.2 \%)$ patients exhibited non-severe symptoms with an $\mathrm{OSCl}<6$, while $271(4.8 \%)$ exhibited severe symptoms with an $\mathrm{OSCl} \geq 6$.

\subsection{Model performance}

The cross-validated AUROC of the XGBoost and LR models were 0.965 (95\% Cl: 0.958-0.972) and 0.938 (95\% Cl: $0.911-0.959)$, respectively $(p=0.039)$. We chose the XGBoost model as our baseline since it outperformed the LR across all performance measures. Regarding AUROC, we also examined XGBoost's outperformance across four different severity endpoints (Additional file 1: Table S2).

\subsection{Model interpretability}

According to SHAP, age and lymphocyte count were the most important risk factors for predicting OSCI $\geq$ 6 (Fig. 3a). Patient age, lymphocyte count, platelet count, BMI, hematocrit, and heart rate all exhibited non-linear influences in predicting disease severity (Fig. $3 b$ ). In addition to the overall impact of each feature on the model's output, SHAP provides patient-specific influences of each variable on their predicted disease severity (Additional file 1: Fig. S1).

\subsection{Predictive performance under limited data availability}

An AUROC of 0.965 (95\% Cl: $0.958-0.972)$ was obtained with the baseline model $(F+L+)$, which included all 37 variables. Notably, a reduction in its performance was found to be insignificant when 20 variables were eliminated, resulting in the F- $\mathrm{L}+$ model (Fig. 4a). The F $+\mathrm{L}+$ model achieved both sensitivity and specificity of greater than $90 \%$. The F-L + model achieved a sensitivity of $88 \%$ and a PPV of $31 \%$. The F-L + model still outperformed the LR model regarding all performance measures.

The F + L- model obtained an AUROC of 0.946 (95\% Cl: 0.936-0.956), which included 32 variables (Additional file 1: Table S3). The reduction in performance was found to be insignificant when 21 variables were eliminated, resulting in the F-L-model (Fig. 4b). The F + L- and F-L-models achieved sensitivities of $84 \%$ and $81 \%$, respectively (Table 1). Differences in AUROCs were observed when laboratory variables were excluded, both in the full and reduced models, which implied that the laboratory variables have a solid discriminative power (all $p \leq 0.01$ ). The AUROCs of all hold-out sets were statistically indifferent from the cross-validation results, inferring the model's generalizability to unseen data (Additional file 1: Table S4). Detailed results and the selected variables used at each step of the RFE are presented in Additional file 1: Tables S3 and S5. 
Table 1

Comparison of model performances.

\begin{tabular}{|c|c|c|c|c|c|c|c|}
\hline Model & $\begin{array}{l}\text { Number of } \\
\text { variables }\end{array}$ & AUROC & Specificity & Sensitivity & Accuracy & PPV & NPV \\
\hline \multirow[t]{2}{*}{$\mathrm{F}+\mathrm{L}+$} & 37 & 0.965 & 0.900 & 0.905 & 0.900 & 0.314 & 0.995 \\
\hline & & $\begin{array}{l}(0.958- \\
0.972)\end{array}$ & $\begin{array}{l}(0.892- \\
0.909)\end{array}$ & $\begin{array}{l}(0.868- \\
0.942)\end{array}$ & $\begin{array}{l}(0.892- \\
0.908)\end{array}$ & $\begin{array}{l}(0.295- \\
0.335)\end{array}$ & $\begin{array}{l}(0.993- \\
0.997)\end{array}$ \\
\hline \multirow[t]{2}{*}{$\mathrm{F}-\mathrm{L}+$} & 17 & 0.963 & 0.900 & 0.884 & 0.899 & 0.309 & 0.994 \\
\hline & & $\begin{array}{l}(0.955- \\
0.971)\end{array}$ & $\begin{array}{l}(0.892- \\
0.908)\end{array}$ & $\begin{array}{l}(0.839- \\
0.921)\end{array}$ & $\begin{array}{l}(0.891- \\
0.907)\end{array}$ & $\begin{array}{l}(0.289- \\
0.329)\end{array}$ & $\begin{array}{l}(0.991- \\
0.996)\end{array}$ \\
\hline \multirow[t]{2}{*}{$F+L-$} & 32 & 0.946 & 0.900 & 0.839 & 0.897 & 0.297 & 0.991 \\
\hline & & $\begin{array}{l}(0.936- \\
0.956)\end{array}$ & $\begin{array}{l}(0.891- \\
0.908)\end{array}$ & $\begin{array}{l}(0.793- \\
0.884)\end{array}$ & $\begin{array}{l}(0.888- \\
0.905)\end{array}$ & $\begin{array}{l}(0.276- \\
0.319)\end{array}$ & $\begin{array}{l}(0.988- \\
0.994)\end{array}$ \\
\hline \multirow[t]{2}{*}{ F-L- } & 11 & 0.942 & 0.901 & 0.810 & 0.896 & 0.291 & 0.989 \\
\hline & & $\begin{array}{l}(0.931- \\
0.953)\end{array}$ & $\begin{array}{l}(0.892- \\
0.909)\end{array}$ & $\begin{array}{l}(0.756- \\
0.860)\end{array}$ & $\begin{array}{l}(0.888- \\
0.904)\end{array}$ & $\begin{array}{l}(0.270- \\
0.313)\end{array}$ & $\begin{array}{l}(0.987- \\
0.992)\end{array}$ \\
\hline $\begin{array}{l}\text { AUROC } \\
\text { positiv } \\
\text { laborat } \\
\text { data }\end{array}$ & $\begin{array}{l}\text { area under } \\
\text { edictive va } \\
\text { data, F+L }\end{array}$ & $\begin{array}{c}+L+=1 \\
\text { model }\end{array}$ & $\begin{array}{l}\text { odel with I } \\
\text { sut laborat }\end{array}$ & $\begin{array}{l}\text { tic, NPV } \\
\text { ratory dat } \\
\text { data, F-L- }\end{array}$ & duced $m$ & $\begin{array}{l}\text { ive value } \\
\text { d model } \\
\text { I without }\end{array}$ & $\begin{array}{l}\mathrm{V}= \\
\text { oratory }\end{array}$ \\
\hline
\end{tabular}

\subsection{Optimal triage under limited resource availability}

We performed simulations using patient flow data that was generated using the SIR model with varying ROs. The overall DES workflow is illustrated in Fig. 5. The DES using hypothetical patient influx scenarios revealed that the optimal threshold ranges from 0.02 to 0.66 , while the respective minimized mortality rates ranged from $0.017(1.7 \%)$ to $0.042(4.2 \%)$ (Fig. 6). The optimal threshold values and minimized mortality rates for each R0 show that a larger R0 value tends to result in increases in both of these variables. The optimal threshold is increased along with the $\mathrm{R} 0$ values to increase precision for severe patients while fully utilizing the ICU. The optimized mortality rates were increased due to increased proportion of death outside ICU followed by a larger volume of patient influx. The benefits of utilizing an optimal triage threshold are clear when compared with the conventional Youden Index (J-index) as a benchmark value, which was 0.013 . Decreased mortality rates (= $\left.\frac{\mathrm{J} \text {-indexmortalityrate - optimizedmortalityrate }}{\mathrm{J} \text {-indexmortalityrate }}\right)$ were notably large in a magnitude ranging from $6.1-18.1 \%$.

We observed a convex relationship of mortality rates in accordance with the thresholds (Fig. 7). The mortality rate was minimized at a point where the proportion of type I deaths, which has the lowest $P_{\text {death }}$ $(50.7 \%)$, was maximized. For example, when R0 was 1.5 , the proportion of type I deaths was maximized at the optimal threshold, accounting for $66.4 \%$ of total deaths. However, a threshold that is too low leads 
limited capacity for actual severe patients then decreases the proportion of type I deaths and increases those of type II deaths. Conversely, a threshold that is too high would result in unnecessary rejection for severe patients, which then decreases the proportion of type I deaths and increases those of type III deaths.

In situations of excessively high RO values and increased ICU demands, increasing the triage threshold to reject more patients will still deplete the ICU capacity. Therefore, adjusting the threshold will mostly result in trade-offs between the number of threshold- and capacity-dependent rejections, limiting the influence of threshold adjustment on minimizing patient mortality. In situations of sufficiently low R0 values, the effect of threshold optimization is reduced along with its necessity. Nonetheless, the large reduction in mortality rates among the remaining influxes outlines the substantial benefits of optimizing the patient triage threshold under resource constraints.

\section{Discussion}

A distinctive feature of our $\mathrm{F}+\mathrm{L}+$ model is its high discriminative power with an AUROC that exceeded 0.97 in both the cross-validation and hold-out settings. Previous prediction models for determining the clinical deterioration of COVID-19 patients have reported accuracies between 0.77 and 0.91 [2-5]. Additionally, these models require specific diagnostic data, including laboratory data, peripheral oxygen saturation, or radiographic findings, to maintain their accuracies. Moreover, to what extent these models' performance abilities are maintained during the partial absence of data has not been studied. For our $\mathrm{F}+$ $\mathrm{L}+$ model to be effectively implemented in practice, we confirmed that our reduced models maintained an adequate discriminative power even in the partial absences of data. The advantages of our reduced models include not only their generalizability to unseen data, but also their applicability within scenarios wherein there is limited clinical data. As timely triage is important for COVID-19 patients, our reduced models can be utilized during the early triage stages at a patient's arrival while waiting for the $\mathrm{F}+\mathrm{L}+$ model to more accurately stratify their disease severity risk. Given the acute exacerbation of pneumonia in COVID-19 patients, our model can also be used to re-evaluate hospitalized patients in the short-term, so that those whose clinical manifestations are likely to exacerbate can be early identified [19].

A noteworthy feature of our model is its ability to discriminate between patient-specific contributing factors for disease exacerbation and their individual contributions using SHAP values. Current COVID-19 treatment guidelines provide recommendations based on the average-risk patient under limited available insights into their disease stage [10]. These recommendations provide a one-size-fits-all approach to all patients, which is problematic for those with more complex or atypical disease presentations. Our model obviates the need for arbitrary patient risk groupings and is, therefore, useful in maximizing their survival odds based on individual risk stratification. Furthermore, our models can be integrated into electronic medical record systems, which utilize coding algorithms, as a notification system that helps in the early identification of disease exacerbation risk factors. 
The validity of our model is supported by the high consistency between the results of its interpretation using SHAP, and previously reported prognosticators of COVID-19 severity [20-25]. We noted that old age, followed by lymphopenia and thrombocytopenia, exhibit the highest Shapley values for disease exacerbation. We presumed older age interacts with relevant features in older adults, including poor functional performances and increased frailty, which are associated with adverse outcomes and increased mortality among patients with respiratory syndromes [26]. Our findings also add to the literature that lymphopenia plays an important role in COVID-19 exacerbation [22-25]. Lymphopenia is exerted by the lowering of lymphocytes due to injured alveolar epithelial cells and is commonly observed in COVID-19 patients [27]. Consistent with previous studies, thrombocytopenia was also found to be associated with adverse COVID-19 outcomes $[23,28]$. It has been suggested that a reduction or morphological alternation of the pulmonary capillary bed exerts pathological platelet defragmentation because the lung is a platelet release site with mature megakaryocytes [29]. Our prediction model supports the notion that early identification of COVID-19 exacerbation, before a hematological crisis occurs, is necessary for ensuring a better prognosis.

There is no existing study that examines COVID-19 severity prediction models that provides an explicit solution for the delivery of optimal triage using threshold modification that accounts for limited resource availability. We conducted a DES on our F-L + model to examine its discrimination thresholds that are usable in an adaptive manner across various patient influx scenarios and the related ICU availability. Our simulations reveal that applying the optimal thresholds will minimize the mortality rate of each patient influx scenario. Our hypothesis is supported by the significant differences found in mortality rates between the J-index and our optimized thresholds when applied to the expected influx volumes. This observation implies the potential or our model to substantially reduce COVID-19 mortality rates through its appropriate adjusting of triage thresholds.

A limitation of our study was its incorporation of a single, national cohort of Asian ethnicity, which impacts our findings' generalizability. An external validation using a more multi-ethnic population is thus needed to determine if a similar discrimination performance occurs among other ethnic groups. However, to ensure our model's robustness, we implemented 10 -fold cross-validation with additional confirmation using the hold-out cohort. Another limitation was that the triage threshold was evaluated using a simulation. Simulations do not yield concrete answers, nor are they able to assess all kinds of potential situations [30].

\section{Conclusions}

We developed and validated a robust prediction model, with an explanatory feature, that enhances the efficiency of COVID-19 triage. Our model has the potential for effective use because it can be integrated into electronic medical record systems that utilizes coding algorithms. We further proposed a novel, adaptive triage model that utilizes patient influx volumes according to concurrent ICU capacity to establish the optimal thresholds for triage strategies. Our study reveals that COVID-19 treatment plans

Loading [MathJax]/jax/output/CommonHTML/fonts/TeX/fontdata.js ext expertise to guarantee maximum efficacy. 


\section{Declarations}

Ethics approval: This study was approved by an institutional ethics committee (2020-0883-001) and the Korea Disease Control and Prevention Agency (KDCA) epidemiological survey and analysis committee (20201120_4a).

Consent to participate: Informed consent was not required because the study did not modify the patient's management and the data were anonymously collected.

Availability of data and material: Data supporting this study's findings are available from the corresponding author upon reasonable request. A portion of the study data is available within Additional file 1. The original dataset is not publicly available due to the KDCA's confidentiality policy.

Competing interests: All authors have completed the ICMJE uniform disclosure form at www.icmje.org/ coi_disclosure.pdf and declare: all authors had financial support from the Infection Prevention Strategy Development Program of Korea for the submitted work; no financial relationships with any organizations that might have an interest in the submitted work in the previous three years; no other relationships or activities that could appear to have influenced the submitted work.

Funding: This study was supported by the Infection Prevention Strategy Development Program of Korea (HW20C2103). The funding source had no role in the study design; analysis and interpretation of the data; in the writing of the manuscript; and the decision to submit the article for publication.

Author's contributions: J.M.K. and H.K.L. contributed towards the conception, acquisition, analysis, interpretation of the study data, and writing of the original draft. K.H.L. and K.S.L. contributed towards the interpretation of the study data. J.A. contributed towards the review, editing, and supervision. K.C.K. contributed towards the conception and writing of the original draft, review, editing, and supervision. All authors read and approved the final manuscript.

Acknowledgments: We would like to thank the Korea Disease Control and Prevention Agency (KDCA) and the Health Information Managers in the participating hospitals for their assistance in collecting the medical records utilized in this study. This study was supported through the Infection Prevention Strategy Development Program of Korea (HW20C2103).

Code availability: The code used to develop and evaluate this study's models is available at https://github.com/minkim88/Optimal-Triage-COVID-19

\section{References}

1. World Health Organization. Critical preparedness, readiness and response actions for COVID-19: interim guidance, 7 March 2020. World Health Organization.

https://apps.who.int/iris/handle/10665/331422. Accessed 1 June 2021. 
2. Gupta RK, Harrison EM, Ho A, et al. Development and validation of the ISARIC 4C Deterioration model for adults hospitalised with COVID-19: a prospective cohort study. Lancet Respir Med. 2021;9:34959. https://doi.org/10.1016/S2213-2600(20)30559-2.

3. Mejia-Vilet JM, Córdova-Sánchez BM, Fernández-Camargo DA, et al. A risk score to predict admission to the intensive care unit in patients with Covid-19: the ABC-GOALS score. Salud Publica Mex. 2020. https://doi.org/10.21149/11684.

4. Ji D, Zhang D, Xu J, et al. Prediction for progression risk in patients with COVID-19 pneumonia: the CALL Score. Clin Infect Dis. 2020;71:1393-9. https://doi.org/10.1093/cid/ciaa414.

5. Knight SR, Ho A, Pius R, et al. Risk stratification of patients admitted to hospital with covid-19 using the ISARIC WHO Clinical Characterisation Protocol: development and validation of the 4C Mortality Score. BMJ. 2020;370:m3339. https://doi.org/10.1136/bmj.m3339.

6. Gao Y, Cai G, Fang W, et al. Machine learning based early warning system enables accurate mortality risk prediction for COVID-19. Nat Commun. 2020;11:5033. https://doi.org/10.1038/s41467-02018684-2.

7. Bolourani S, Brenner M, Wang P, et al. A machine learning prediction model of respiratory failure within 48 hours of patient admission for COVID-19: model development and validation. J Med Internet Res. 2021;23:e24246. https://doi.org/10.2196/24246.

8. Guyon I, Weston J, Barnhill S, et al. Gene Selection for Cancer Classification using Support Vector Machines. Mach Learn. 2002;46:389-422. https://doi.org/10.1023/A:1012487302797.

9. Karnon J, Stahl J, Brennan A, et al. Modeling using discrete event simulation: a report of the ISPORSMDM Modeling Good Research Practices Task Force-4. Value Health. 2012;15:821-7. https://doi.org/10.1016/j.jval.2012.04.013.

10. World Health Organization. WHO R\&D Blueprint novel Coronavirus COVID-19 Therapeutic Trial Synopsis. February 18, 2020, Geneva, Switzerland. https://www.who.int/blueprint/prioritydiseases/key-action/COVID-

19_Treatment_Trial_Design_Master_Protocol_synopsis_Final_18022020.pdf. Accessed 1 June 2021.

11. Chen T, Guestrin C. XGBoost: A scalable tree boosting system. In: Proceedings of the 22nd ACM SIGKDD International Conference on Knowledge Discovery and Data Mining [Internet]. New York, NY, USA: ACM; 2016:785-94. https://doi.org/10.1145/2939672.2939785.

12. Lundberg SM, Lee S. A unified approach to interpreting model predictions. In Proceedings of the 31 st International Conference on Neural Information Processing Systems (NIPS'17). Curran Associates Inc., Red Hook, NY, USA; 2017:4768-77.

13. Hethcote HW. The mathematics of infectious diseases. SIAM review, 42.4; 2000:599-653. https://doi.org/10.1137/S0036144500371907.

14. Lee $Y$, Hong C, Kim D, et al. Clinical course of asymptomatic and mildly symptomatic patients with coronavirus disease admitted to community treatment centers, South Korea. Emerg Infect Dis. 2020;26:2346-52. https://doi.org/10.3201/eid2610.201620. 
15. Caflisch R. Monte Carlo and quasi-Monte Carlo methods. Acta Numerica. 1998;7:1-49. https://doi.org/10.1017/S0962492900002804.

16. Wood RM, Pratt AC, Kenward C, et al. The value of triage during periods of intense COVID-19 demand: simulation modeling study. Med Decis Making. 2021;41:393-407. https://doi.org/10.1177/0272989X21994035.

17. Korea Disease Control and Prevention Agency. Infectious Disease Portal. http://www.kdca.go.kr/npt/biz/npp/portal/npplssuelcdMain.do. Accessed 1 June 2021.

18. ICNARC. ICNARC report on COVID-19 in critical care. 2020. https://www.icnarc.org/OurAudit/Audits/Cmp/Reports. Accessed 1 June 2021.

19. Haimovich AD, Ravindra NG, Stoytchev S, et al. Development and validation of the quick COVID-19 Severity Index: a prognostic tool for early clinical decompensation. Ann Emerg Med. 2020;76:44253. https://doi.org/10.1016/j.annemergmed.2020.07.022.

20. Ye J, Zhang X, Zhu F, et al. Application of a prediction model with laboratory indexes in the risk stratification of patients with COVID-19. Exp Ther Med. 2021;21:182. https://doi.org/10.3892/etm.2021.9613.

21. Hu L, Chen S, Fu Y, et al. Risk factors associated with clinical outcomes in 323 coronavirus disease 2019 (COVID-19) hospitalized patients in Wuhan, China. Clin Infect Dis. 2020;71:2089-98. https://doi.org/10.1093/cid/ciaa539.

22. Lassau N, Ammari S, Chouzenoux E, et al. Integrating deep learning CT-scan model, biological and clinical variables to predict severity of COVID-19 patients. Nat Commun. 2021;12:634. https://doi.org/10.1038/s41467-020-20657-4.

23. Lippi G, Plebani M, Henry BM. Thrombocytopenia is associated with severe coronavirus disease 2019 (COVID-19) infections: A meta-analysis. Clin Chim Acta. 2020;506:145-8. https://doi.org/10.1016/j.cca.2020.03.022.

24. Henry BM, Lippi G. Chronic kidney disease is associated with severe coronavirus disease 2019 (COVID-19) infection. Int Urol Nephrol. 2020;52:1193-4. https://doi.org/10.1007/s11255-020-024519.

25. Ruan Q, Yang K, Wang W, et al. Clinical predictors of mortality due to COVID-19 based on an analysis of data of 150 patients from Wuhan, China. Intensive Care Med. 2020;46:846-8. https://doi.org/10.1007/s00134-020-05991-x.

26. Wu G, Yang P, Xie Y, et al. Development of a clinical decision support system for severity risk prediction and triage of COVID-19 patients at hospital admission: an international multicentre study. Eur Respir J. 2020;56:2001104. https://doi.org/10.1183/13993003.01104-2020.

27. Chan JF, Yuan S, Kok K, et al. A familial cluster of pneumonia associated with the 2019 novel coronavirus indicating person-to-person transmission: a study of a family cluster. Lancet. 2020;395:514-23. https://doi.org/10.1016/S0140-6736(20)30154-9.

28. Goodall JW, Reed TAN, Ardissino M, et al. Risk factors for severe disease in patients admitted with Loading [MathJax]/jax/output/CommonHTML/fonts/TeX/fontdata.js ective cohort study. Epidemiol Infect. 
2020;148:e251. https://doi.org/10.1017/S0950268820002472.

29. Yang M, Ng MH, Li CK. Thrombocytopenia in patients with severe acute respiratory syndrome (review). Hematology. 2005;10:101-5. https://doi.org/10.1080/10245330400026170.

30. Tulsian PC, Pandey V. Quantitative Techniques: Theory \& Problems. New Delhi: Pearson Education, Singapore; 2002.

\section{Figures}

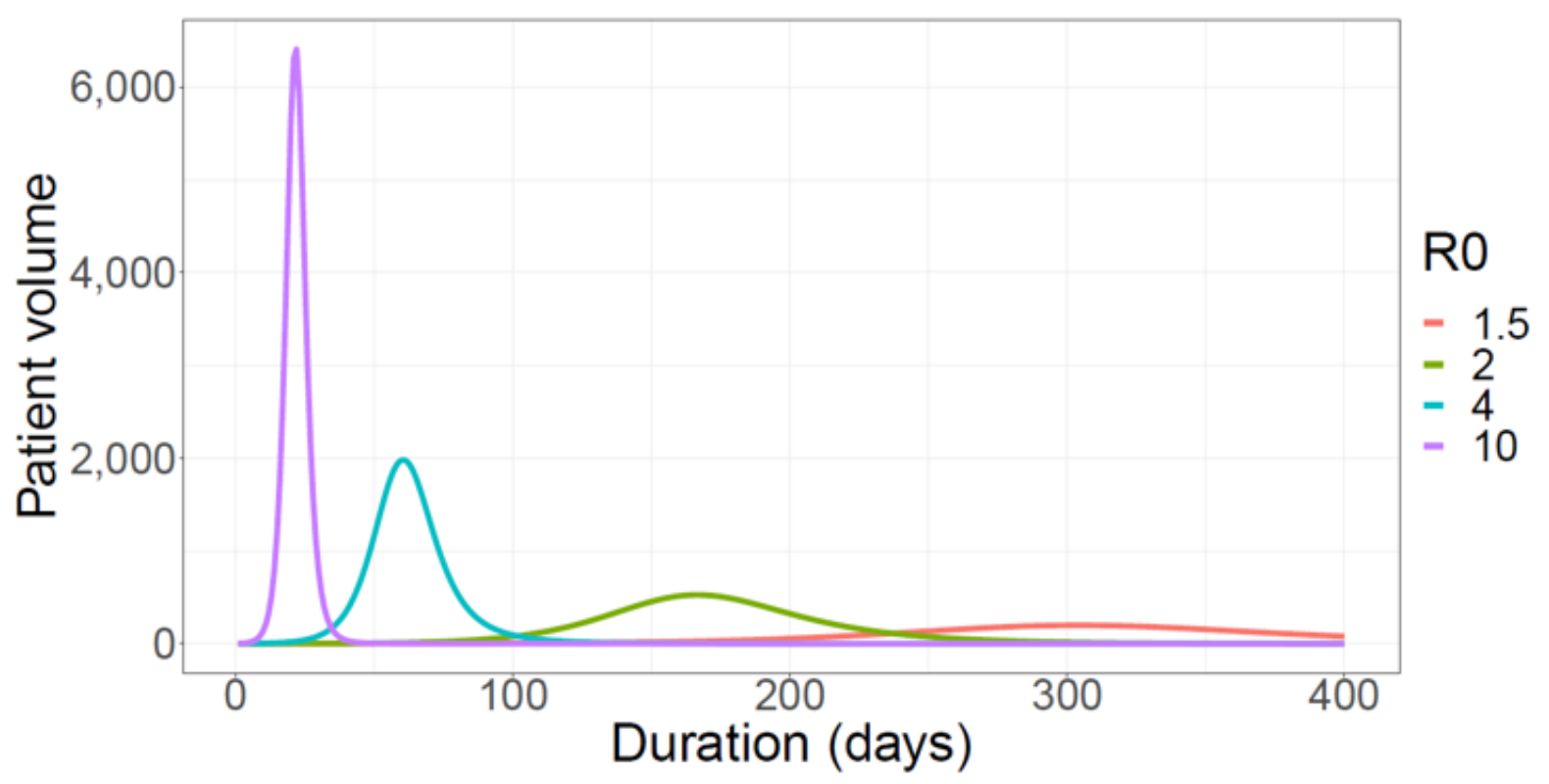

\begin{tabular}{|c|c|c|c|c|}
\hline $\mathbf{R 0}$ & $\mathbf{1 . 5}$ & $\mathbf{2}$ & $\mathbf{4}$ & $\mathbf{1 0}$ \\
\hline \hline Duration (days) & 677 & 416 & 198 & 65 \\
\hline Cumulative patients & 34,965 & 47,802 & 58,799 & 59,993 \\
\hline $\begin{array}{c}\text { Maximum daily patients } \\
(=\text { maximum height) }\end{array}$ & 198 & 526 & 1,980 & 6,407 \\
\hline
\end{tabular}

\section{Figure 1}

Patient influx. SIR simulated COVID-19 patient influx. COVID-19 = coronavirus disease 2019 


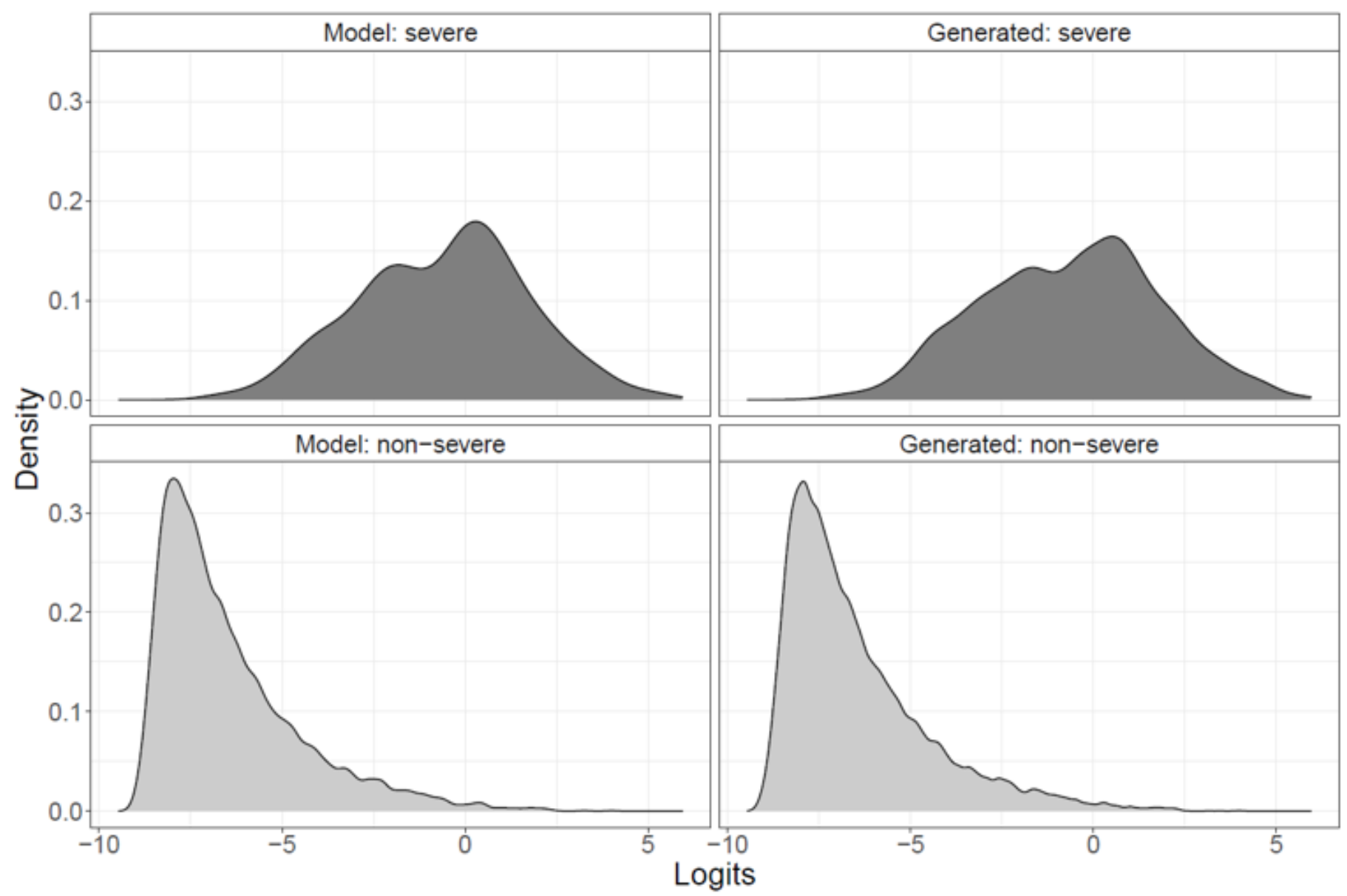

Figure 2

Prediction probability distribution graph of patients in the out-of-fold samples. 

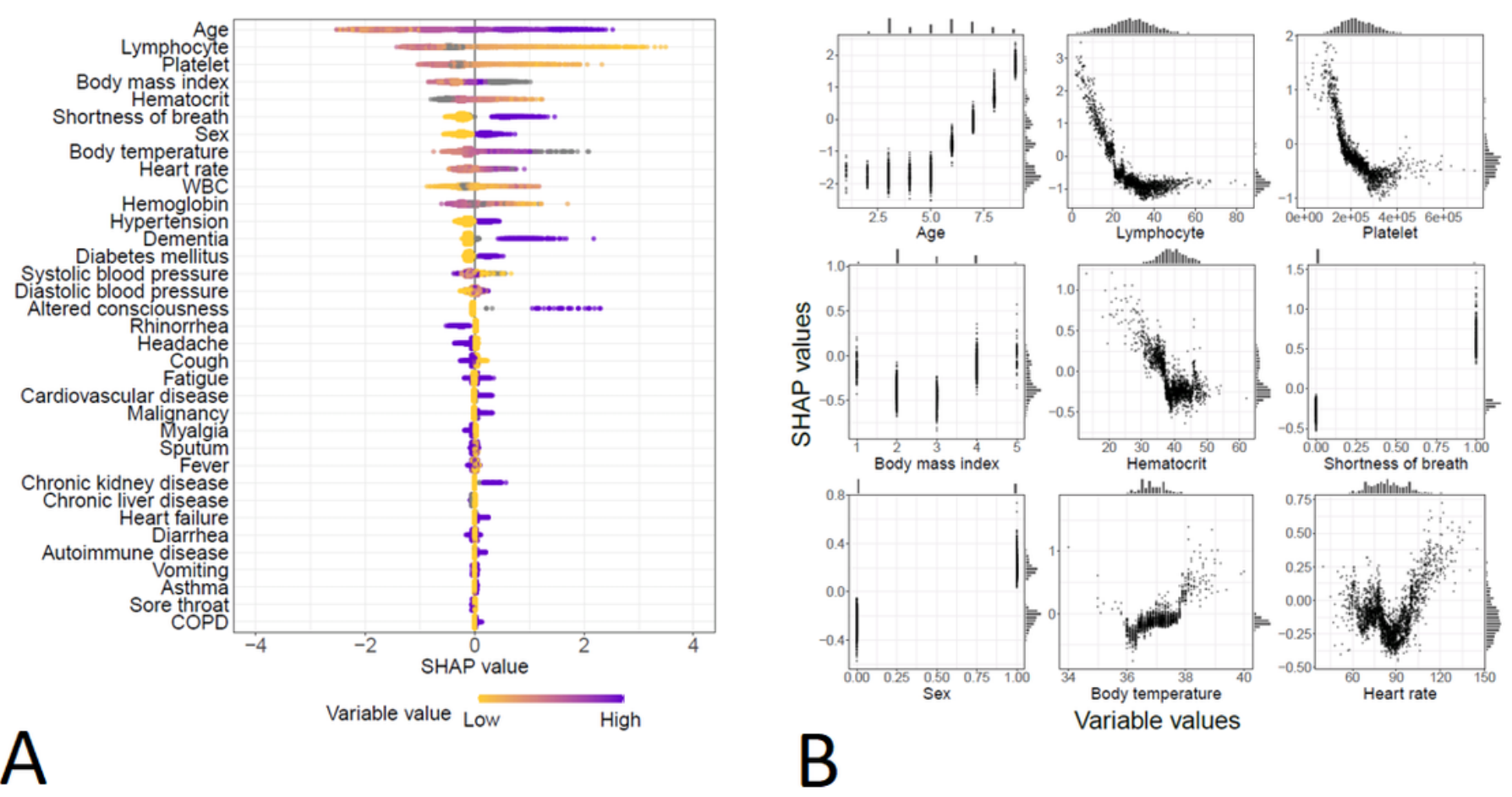

Figure 3

Relationships between each feature and the SHAP values. a. Summary plot in which each dot-point represents the SHAP value of a patient in the dataset used to construct the developed model. The dots are plotted for every feature used to fit the baseline model, excluding two features (pregnancy and number of weeks pregnant) that were not selected for the developed model. The SHAP values are displayed in rank order, based on their feature importance, on the y-axis as calculated by averaging the absolute SHAP values of each dot. The points' location on the x-axis shows their impact on the predictive output of the model. Purple indicates a relatively high feature value, while yellow represents a relatively low feature value. Grey-colored dots represent missing values. b. Dependence plots for each of the top nine important features, including: patient age, lymphocyte count, platelet count, BMI, hematocrit, shortness of breath, sex, body temperature, and heart rate. Each scatter plot shows the impact of each feature on the predictions made by the study model. The $x$-axis represents the variables' values and the $y$ axis represents their SHAP values. The inflection points indicate the non-linear impact of a feature on the model's prediction. $\mathrm{BMI}=$ body mass index, SHAP = Shapley additive explanations 


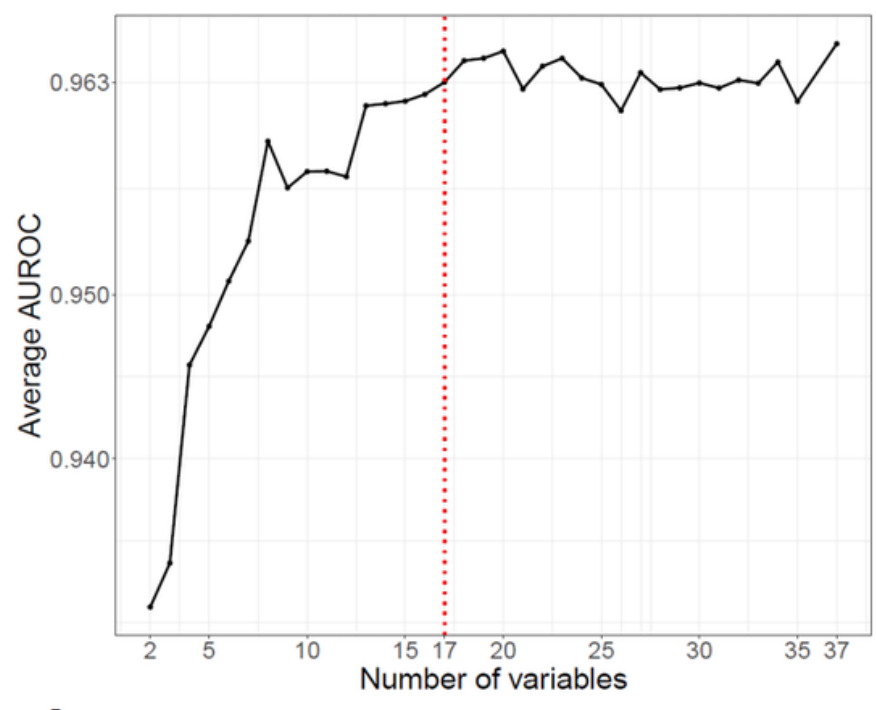

A

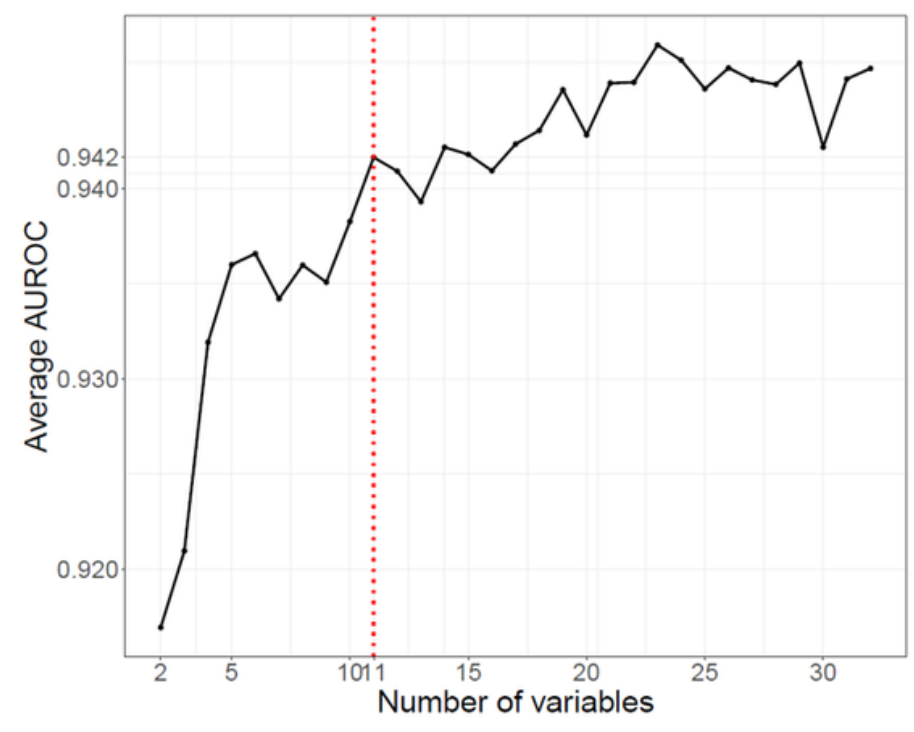

B

\section{Figure 4}

Changes to the model's performance after applying the RFE. The outcome of the entire RFE was presented in terms of its AUROC. Each point represents the performance of our model after the lowestranked feature had been eliminated. a. The AUROC of the model, including the laboratory variables. An RFE was performed on the $\mathrm{F}+\mathrm{L}+$ model set that consists of 37 variables, including five laboratory ones. The $\mathrm{F}+\mathrm{L}+$ model during the first iteration was developed using 37 variables, while the $\mathrm{F}-\mathrm{L}+$ model was developed using 17 variables. In the $F+L+$ model, pregnancy and pregnancy week variables showed zero SHAP values; hence, these variables were eliminated. b. The AUROC of the model excluding the laboratory variables. The RFE was performed on the $\mathrm{F}+\mathrm{L}$ - model set that consists of 32 variables, in which the five laboratory variables were excluded. The $\mathrm{F}+\mathrm{L}$ - model at the first iteration was developed based on 32 variables, while the F-L- model was developed using 11 variables. AUROC = area under the receiver operating characteristic, RFE = recursive feature elimination, $\mathrm{F}+\mathrm{L}+=$ full model with laboratory data, $\mathrm{F}-\mathrm{L}+$ $=$ reduced model with laboratory data, $\mathrm{F}+\mathrm{L}-=$ full model without laboratory data, $\mathrm{F}-\mathrm{L}-=$ reduced model without laboratory data 


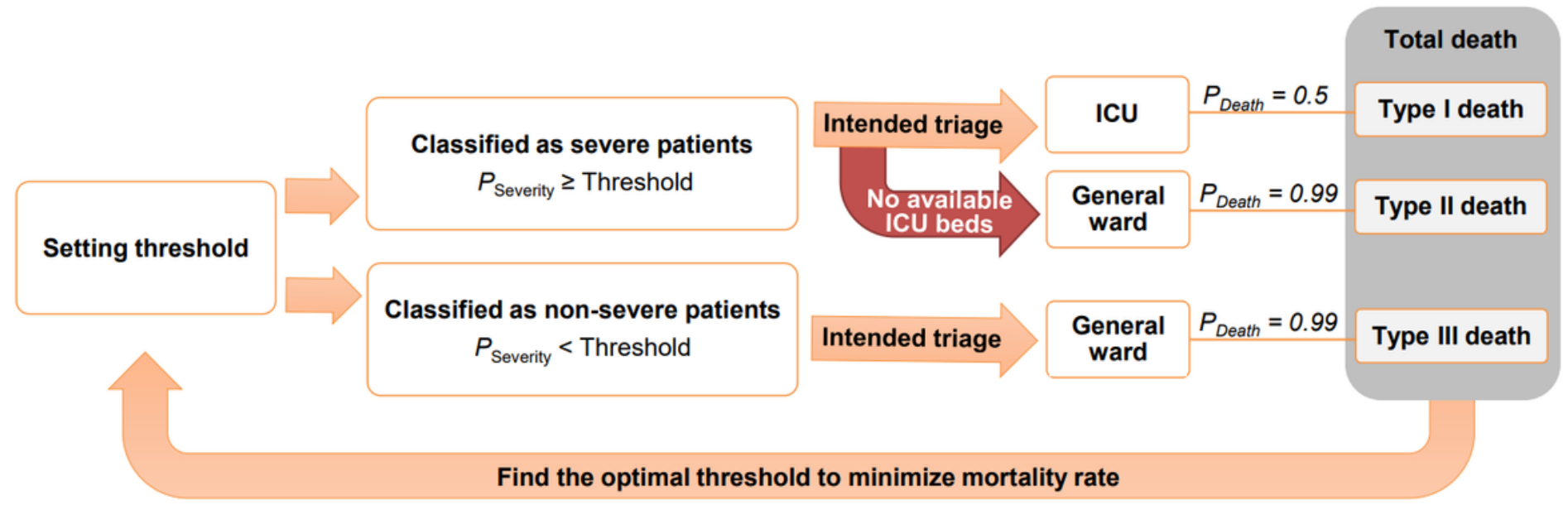

\section{Figure 5}

Please see the Manuscript file for the complete figure caption. 


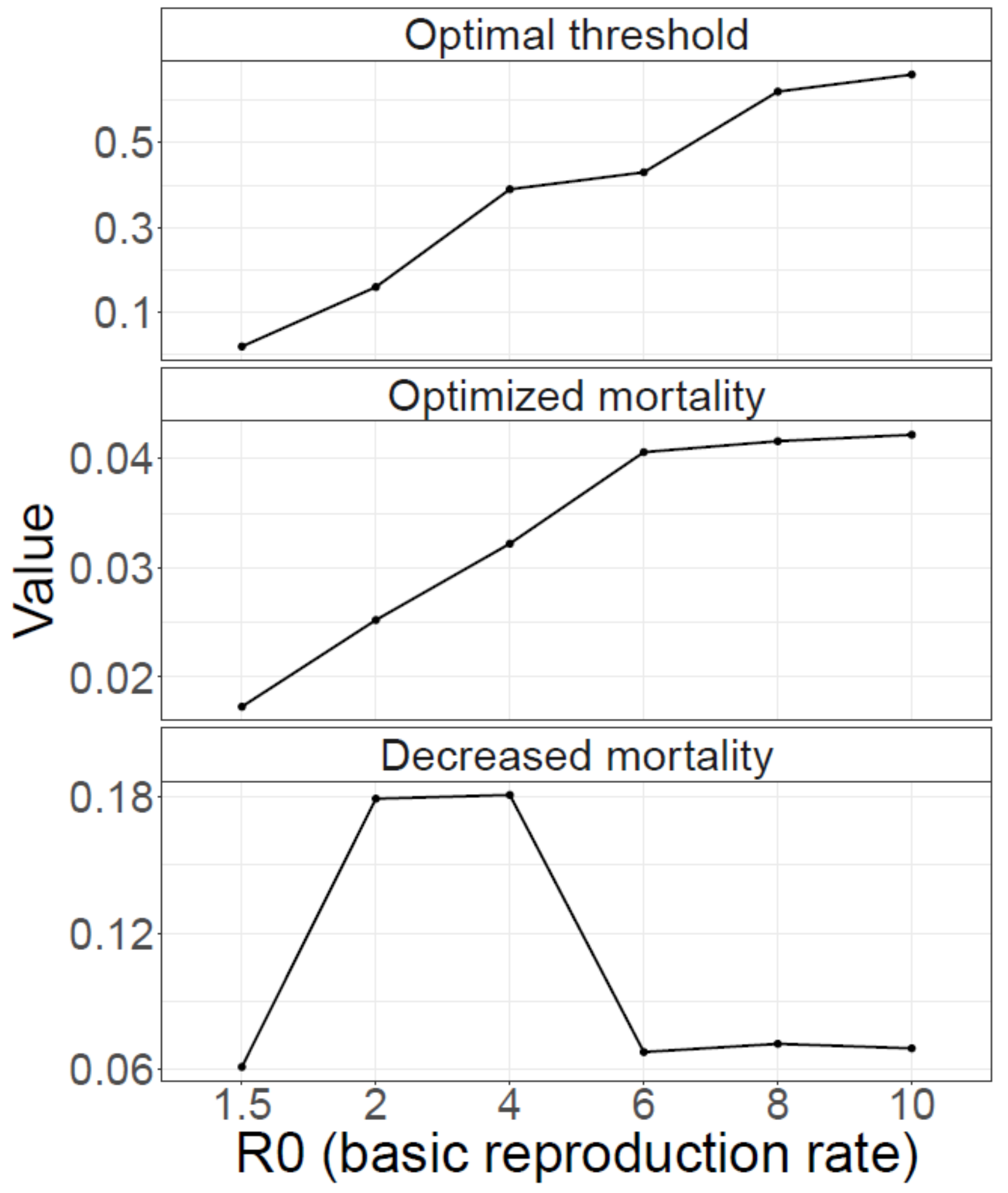

Figure 6

Optimized results of the patient triage simulations. Hypothetical influx simulation results. R0 = basic reproduction rate, decreased mortality rate $=(\mathrm{J}$ index mortality rate - optimized mortality rate) $/ \mathrm{J}$ index mortality rate. 


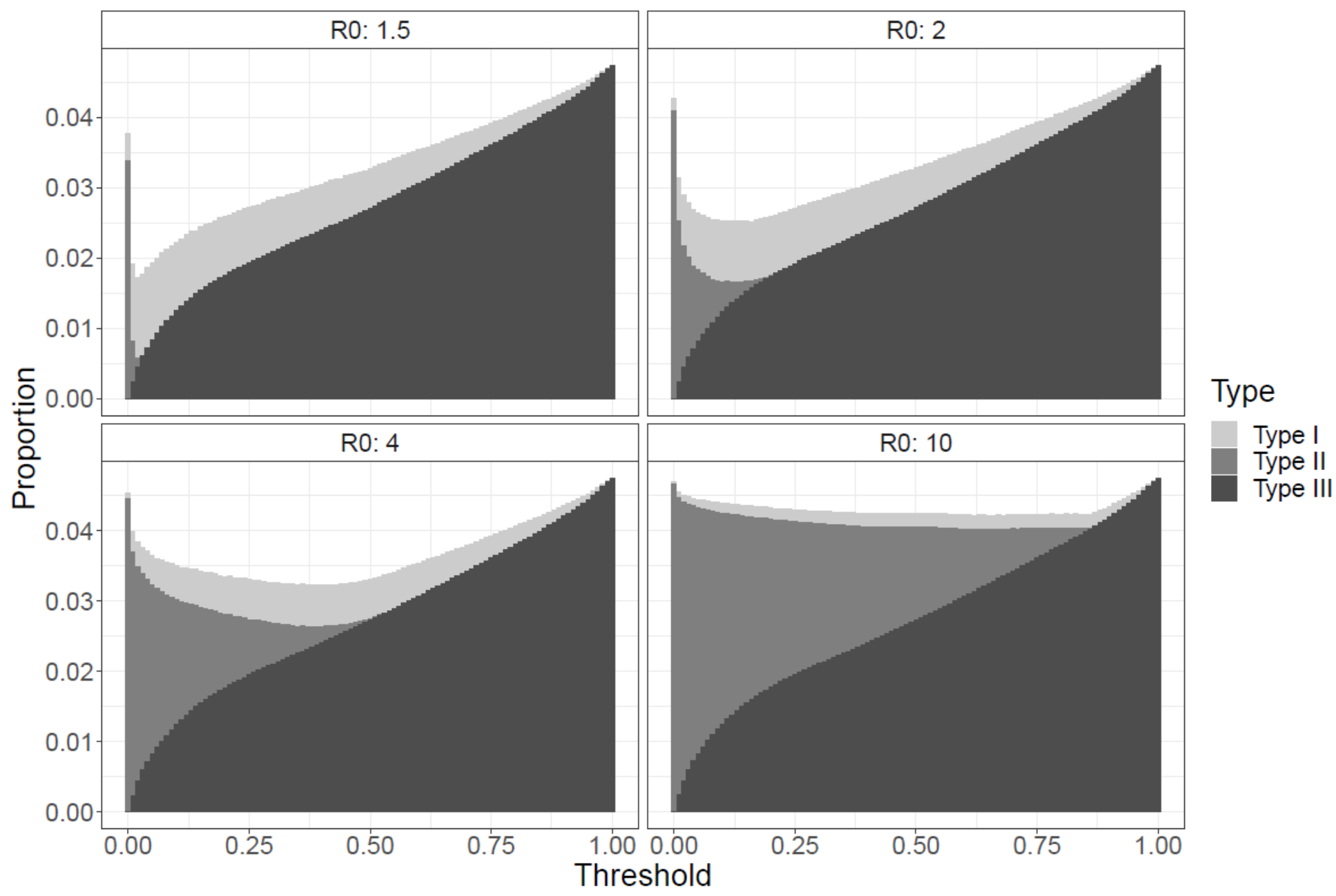

Figure 7

Please see the Manuscript file for the complete figure caption.

\section{Supplementary Files}

This is a list of supplementary files associated with this preprint. Click to download.

- Additionalfile1.docx 